\title{
Economic burden of acute steroid-refractory graft-versus-host disease in commercially insured pediatric patients
}

Michael Grabner, PhD; Eric Strati, PharmD, MBA; Karen Sandman, PhD; and Anna Forsythe, PharmD, MSc, MBA

\section{What is already known about this subject}

- Acute graft-versus-host disease (aGVHD) is a potentially lifethreatening complication of hematopoietic stem cell transplantation (HSCT) often treated with steroids, but there is no standard of care for the more than $50 \%$ of patients who become steroid refractory.

- Steroid-refractory (SR) aGVHD is associated with significantly worse pediatric health outcomes and with economic burden thought to be greater than that seen with steroidresponsive aGVHD.

\section{What this study adds}

- A retrospective analysis of commercial insurance claims data provides insight into the economic burden and health care resource utilization (HCRU) of these pediatric patients across multiple treatment centers.

- Pediatric patients with SR aGVHD experienced more frequent complications and incurred significantly higher HCRU and direct medical costs within the 12 months following alloHSCT than controls who had no claims for GVHD.

- Within 12 months of transplant, the costs incurred by patients with SR aGVHD who ultimately died were significantly higher than for those who survived.

Research Database (study period January 1, 2006-May 31, 2019). Included patients had at least 1 claim for allogeneic HSCT (earliest HSCT claim set as index date), no claims for autologous HSCT, and no pre-index GVHD. Patients were aged less than 18 years with no minimum pre- or post-index continuous enrollment. The GVHD cohort included patients with at least 1 claim for aGVHD over 100 days from index with at least 1 claim for any steroid and at least 1 claim for secondline therapy, both on or after the date of the first aGVHD claim. Patients post-HSCT with no GVHD claims over follow-up formed the comparison cohort. Health care resource utilization and costs over 12 months from the index date were calculated and compared between cohorts using parametric testing.

\section{Author affiliations}

Michael Grabner, PhD, HealthCore, Inc., Wilmington, DE. Eric Strati, PharmD, MBA, Mesoblast, Inc., New York, NY. Karen Sandman, PhD, and Anna Forsythe, PharmD, MSc, MBA, Purple Squirrel Economics, New York, NY.

AUTHOR CORRESPONDENCE: Anna Forsythe, 646.478.8213; annaforsythe@pshta.com

J Manag Care Spec Pharm. 2021;27(5):607-14

Copyright $@ 2021$, Academy of Managed Care Pharmacy. All rights reserved.

RESULTS: 38 patients with SR aGVHD and 184 controls were included. Mean age and sex were similar for aGVHD (8.6 years, 50\% female) and control ( 8.2 years, $45 \%$ female). During the 12-month post-index follow-up, SR aGVHD patients had higher rates of complications vs controls ( ${ }^{*}$ for $P<0.05$ ): anemia (79\% vs 68\%), drug-induced anemia* $(53 \%$ vs $34 \%$ ), neutropenia ( $63 \%$ vs $53 \%$ ), thrombocytopenia ( $58 \%$ vs $42 \%$ ), gastrointestinal complications ${ }^{\star}$ ( $95 \%$ vs $65 \%$ ), and infections ${ }^{\star}$ ( $95 \%$ vs $79 \%)$. Mean inpatient length of stay was longer by 31.6 days $(P<0.01)$ with a total average of 96.0 days for those with SR aGVHD vs 64.3 days for the controls. More SR aGVHD patients required inpatient total parenteral nutrition ( $71 \%$ vs $58 \%$ ), readmission within 12 months of discharge from index hospitalization* (89\% vs $60 \%$ ), ER visits 
( $34 \%$ vs $24 \%$ ), and outpatient visits ( $100 \%$ vs $86 \%$ ). Total 12 -month mean medical costs were higher in aGVHD patients: $\$ 1,212,944$ vs $\$ 673,491(P<0.001)$, mostly because of complication-related costs: $\$ 868,966$ vs $\$ 396,757(P<0.001)$. Among patients with SR aGVHD, mean total costs were higher by about $\$ 1.8$ million $(\$ 2,609,445$ vs $\$ 812,385 ; P=0.014)$ for those who died compared with those who were alive within 12 months.

CONCLUSIONS: SR aGVHD in pediatric patients following HSCT is associated with incremental 12-month medical costs of greater than $\$ 500,000$, driven largely by complications.

Allogeneic hematopoietic stem cell transplantation (alloHSCT) is a potentially curative treatment for children with serious hematologic conditions, including malignancies (eg, acute myeloid leukemia, acute lymphoblastic leukemia, and myelodysplastic syndromes) and nonmalignant disorders (eg, aplastic anemia, sickle cell disease, and thalassemia). ${ }^{1-3}$ However, after allo-HSCT, many patients develop acute graft-versus-host disease (aGVHD), a potentially lifethreatening complication that typically occurs within 100 days of transplantation. ${ }^{4}$

aGVHD is an immunologically mediated response that occurs when donor T-cells recognize antigens on host cells and target them for attack. ${ }^{5}$ Disease manifestations largely involve the skin, gastrointestinal (GI), and liver organ systems. ${ }^{4,6}$ The most commonly occurring symptoms are maculopapular rash, GI disturbances (eg, diarrhea and GI pain), cholestatic hepatitis, and elevated hepatic enzymes, which reflect the tissue damage in these 3 organ systems. ${ }^{4,6,7}$ The complications associated with aGVHD result in substantial morbidity, reduced quality of life, and increased risk of mortality for patients. ${ }^{6,8,9}$

In the United States, 106,112 allo-HSCTs were performed between 2013 and 2017, of which 35\%-50\% of patients were estimated to have developed aGVHD.,10 The incidence of aGVHD varies based on the degree of human leukocyte antigen (HLA)-disparity between the donor and transplant recipient and increases with greater levels of disparity. ${ }^{6,7}$ In children receiving HLA-matched HSCT, the incidence of aGVHD has been reported at $31 \% .{ }^{11}$ In transplant recipients with 1 antigen mismatch, an incidence rate of $75 \%$ has been reported, rising to $90 \%$ in those with $2-3$ antigen mismatches. ${ }^{6}$

Over $50 \%$ of patients who develop aGVHD following alloHSCT are refractory to first-line treatment with steroids, and up to $75 \%$ of patients require additional therapy, ${ }^{6,7}$ Steroid-refractory (SR) aGVHD is associated with greater symptom severity and poorer outcomes. ${ }^{12}$ Mortality rates as high as $90 \%$ have been reported in adults and pediatric patients with SR aGVHD, who fail to respond to initial steroid therapy. ${ }^{13}$ While first-line treatment with steroids is well established in clinical guidelines, there is no consensus for the second-line treatment for SR aGVHD in adult and pediatric patient populations., ${ }^{714-16}$ Therapies used in the second-line setting vary in clinical practice; while patients may undergo extracorporeal photopheresis or may receive drugs such as antibodies targeting tumor necrosis factor alpha or interleukin-2 receptor, inhibitors of mammalian target of rapamycin, mycophenolate mofetil, or alemtuzumab, their use is supported by limited evidence.,14,15 To date, ruxolitinib is the only treatment approved in the United States for SR aGVHD, which is indicated for patients aged 12 years and older, although the clinical trial included only adults. ${ }^{17}$

The economic burden of aGVHD is high. A systematic literature review reported health care costs in the United States ranging from $\$ 114,698$ to $\$ 224,000$ per year in pediatric patients with aGVHD post-HSCT, which were driven by hospitalizations. ${ }^{9,18,19}$ Compared with allo-HSCT pediatric patients without aGVHD, those who developed aGVHD incurred significantly higher incremental health care costs by approximately US\$200,000 and had a longer inpatient stay by 41.5 days $(P=0.004)$ during the first year after transplant. ${ }^{20}$ Preliminary data also suggest that health care costs may be substantially higher in pediatric patients with aGVHD who are refractory to first-line treatment with steroids, similar to the trend seen in adults., ${ }^{9,21}$ However, there is limited evidence on the health care costs and resource utilization of aGVHD in this SR pediatric patient population.

Given the lack of economic data available, we conducted a retrospective analysis of medical and pharmacy claims from a US commercial insurance database to evaluate the economic burden of SR aGVHD in pediatric patients.

\section{Methods}

Retrospective analyses were conducted using medical and pharmacy claims data from the HealthCore Integrated Research Database (HIRD) from January 1, 2006 to May 31, 2019. Claims for aGVHD were assessed using the International Classification of Diseases, Ninth/Tenth Revision, Clinical Modification (ICD-9/10-CM) codes. ICD9/10-CM, Generic Product Identifier, Current Procedural Terminology, and Healthcare Common Procedure Coding System codes were also used for allo-HSCT, autologous stem cell transplant, GVHD, infectious and noninfectious stem cell transplant complications, medications, and total 


\section{TABLE 1}

\begin{tabular}{|c|c|c|}
\hline & $\begin{array}{c}\text { SR aGVHD } \\
n=38\end{array}$ & $\begin{array}{c}\text { Control } \\
\text { (no GVHD) } \\
n=184\end{array}$ \\
\hline Female, n (\%) & $19 \quad(50)$ & $83 \quad(45)$ \\
\hline Age at index (years), mean (SD) & $8.6(5.97)$ & $8.20(5.57)$ \\
\hline \multicolumn{3}{|l|}{ Age categories (years), n (\%) } \\
\hline $0-5$ & $15 \quad(39)$ & (33) \\
\hline $6-11$ & $10 \quad(26)$ & $(32)$ \\
\hline $12-17$ & $13 \quad(34)$ & $(35)$ \\
\hline \multicolumn{3}{|l|}{ Geographic region, n (\%) } \\
\hline Northeast & (13) & (24) \\
\hline Midwest & $4 \quad(11)$ & $(27)$ \\
\hline South & $(42)$ & $(25)$ \\
\hline West & $12 \quad(32)$ & $(21)$ \\
\hline Unknown & (3) & (3) \\
\hline
\end{tabular}

${ }^{a}$ Demographic characteristics were assessed on index HSCT procedure claim. aGVHD = acute graft-versus-host disease; HSCT = hematopoietic stem cell transplant; $S R=$ steroid refractory.

parenteral nutrition (Supplementary Tables 1 to 8, available in online article). Revenue codes were used to assess intensive care unit costs and length of stay (Supplementary Table 9 , available in online article).

Patients included in this study had at least 1 claim for allo-HSCT (earliest HSCT claim set as index date) in an inpatient setting, no claims for autologous HSCT at any time, and no pre-index GVHD. Patients were aged less than 18 years on the index date and were commercially insured, with no minimum pre- or post-index continuous health plan enrollment. Two patient cohorts were evaluated: those with aGVHD and those without GVHD. The aGVHD cohort included patients with SR aGVHD, defined as those with at least 1 claim for aGVHD over 100 days from the index date and at least 1 claim for any steroid on or after date of first aGVHD claim and at least 1 claim for any second-line therapy (Supplementary Table 7, available in online article) or extracorporeal photopheresis on or after date of the first aGVHD claim.

The comparison cohort included post-HSCT patients without any GVHD claims over their entire available followup period. Complications, health care resource utilization (HCRU), and per-patient costs over 12 months from the

index date were calculated based on claims and compared across cohorts. Selected cost data were also reported in a per-patient per-month (PPPM) format. Outcomes were also assessed over 24 months. Costs are presented as the combined amount paid by the health plan and the patient. Patient out-of-pocket costs included all coinsurance, deductible, copayment, or other patient outlays. Costs were adjusted to 2018 US dollars (USD), using the medical care component of the Consumer Price Index provided by the Bureau of Labor Statistics. ${ }^{22}$ Mortality data from the National Death Index (NDI) were linked to claims for a subset of patients.

\section{STATISTICAL ANALYSIS}

Descriptive statistics including means and SDs were reported for continuous variables, and absolute/relative frequencies were reported for categorical variables. At follow-up, categorical outcomes were compared across the 2 cohorts using chi-square or Fisher's exact tests (without adjustment for differential follow-up time). Generalized linear model regressions with gamma distribution and loglink, weighted for follow-up time, were used to compare continuous variables (hospital length of stay and costs). This study took an exploratory approach, and no adjustment for confounding was conducted.

\section{Results}

\section{PATIENT POPULATION}

A total of 38 pediatric patients who developed SR aGVHD following allo-HSCT and 184 controls without GVHD were included in this study. In 53\% of aGVHD patients, medical claims with codes for chronic GVHD were present during follow-up.

Patient demographics on index date were generally similar between the 2 cohorts. The mean age was 8.6 years for patients with SR aGVHD and 8.2 years for controls without GVHD. Across both cohorts, 33\%-39\% of patients were aged $0-5$ years, and approximately half were female (45\%-50\%; Table 1).

In patients with SR aGVHD, 63\% underwent peripheral blood HSCT compared with $45 \%$ for controls. Median length of follow-up health plan enrollment was 20.5 months and 14.9 months for SR aGVHD patients and controls, respectively. Of the SR aGVHD patients, 25 (66\%) had an enrollment time of at least 12 months, and 15 (39\%) had an enrollment time of at least 24 months. Of the control group, 101 (55\%) had an enrollment time of at least 12 months, and 61 (33\%) had an enrollment time of at least 24 months. Approximately $40 \%$ of patients $(n=16$ SR aGVHD, $n=64$ 


\section{TABLE 2}

\section{Post-HSCT Complications Over 12 Months in Pediatric Patients (<18 Years) with SR aGVHD vs Controls Without GVHD}

\begin{tabular}{l|r|r|c}
\hline & $\begin{array}{c}\text { SR aGVHD } \\
\mathbf{n = 3 8}\end{array}$ & $\begin{array}{c}\text { Control } \\
\text { (no GVHD) } \\
\mathbf{n = 1 8 4}\end{array}$ & P value $^{\mathbf{a}}$ \\
\hline Anemia total, n (\%) & $30(79)$ & $126(68)$ & 0.925 \\
\hline Drug induced $^{\mathrm{b}}$ & $20(53)$ & $62(34)$ & 0.028 \\
\hline Nondrug induced $^{\prime}$ & $24(63)$ & $112(61)$ & 0.792 \\
\hline Neutropenia total, n (\%) & $24(63)$ & $97(53)$ & 0.239 \\
\hline Drug induced & $5(13)$ & $13(7)$ & 0.203 \\
\hline Nondrug induced & $23(61)$ & $95(52)$ & 0.317 \\
\hline Thrombocytopenia total, n (\%) & $22(58)$ & $77(42)$ & 0.070 \\
\hline Liver diseases, n (\%) & $6(16)$ & $31(17)$ & 0.873 \\
\hline Gl diseases, n (\%) & $36(95)$ & $119(65)$ & $<0.001$ \\
\hline Infections, n (\%) & $36(95)$ & $146(79)$ & 0.025 \\
\hline
\end{tabular}

${ }^{a}$ Chi-square test or Fisher test.

${ }^{b}$ Based on ICD-9/10-CM coding. Real-world physician behavior may deviate in some cases, so misclassification is possible.

aGVHD = acute graft-versus-host disease; $G I=$ gastrointestinal; HSCT = hematopoietic stem cell transplant; ICD-9/10-CM= International Classification of Diseases, Ninth/Tenth Revision, Clinical Modification; $S R=$ steroid refractory.

controls) were eligible for mortality linking to the NDI. Over 12 months, all-cause death was observed for $25 \%$ of SR aGVHD patients and 30\% of controls. Over 24 months, all-cause death reached 38\% for SR aGVHD and 33\% for controls.

Patients with SR aGVHD had higher complication rates than controls over the 12-month follow-up period. Significantly higher rates were seen for drug-induced anemia $(53 \%$ vs $34 \%$; P<0.05), GI manifestations $(95 \%$ vs $65 \%$; $\mathrm{P}<0.001)$, and infection $(95 \%$ vs $79 \% ; \mathrm{P}<0.05$; Table 2$)$.

Similar clinical characteristics and complication rates were observed after 24 months follow-up in both cohorts.

\section{HEALTH CARE RESOURCE UTILIZATION}

Length of stay for the inpatient episode surrounding alloHSCT was approximately 60 vs 50 days for SR aGVHD patients vs controls, and readmissions over 12 months after discharge occurred significantly more frequently in the aGVHD cohort (89\% vs $60 \%$; $P<0.001)$. Across all hospitalizations, a similar proportion of patients in both cohorts (45\%-46\%) stayed in the intensive care unit. Patients with SR aGVHD had a numerically longer mean length of stay in intensive care than controls by 16.2 days over 12 months
(36.5 vs 20.3 days) and significantly longer overall hospital length of stay of 31.6 days (96.0 vs 64.3 days; $\mathrm{P}<0.001)$.

Likewise, emergency room (ER) and outpatient visits occurred more frequently in the SR aGVHD cohort (34\% vs $24 \%$ and $100 \%$ vs $86 \%$, respectively; no statistical significance testing was conducted).

Outpatient parenteral nutrition (39\% vs $20 \%$; P $<0.01)$ occurred more frequently in SR aGVHD patients compared with controls.

Within the SR aGVHD cohort, 95\% required second-line treatment over 12 months. Biologic and branded small molecule therapies (etanercept, infliximab, rituximab, ruxolitinib, and tocilizumab) were used by $21 \%$ of patients as second-line options, and 87\% used traditional immunosuppressants, including methotrexate and everolimus.

Similar HCRU and patterns of medication use were observed over the 24-month follow-up period.

\section{DIRECT MEDICAL COSTS}

Mean total 12-month direct medical costs (including the transplant) incurred by patients with SR aGVHD were nearly twice as high compared with controls $(\$ 1,212,944$ vs $\$ 673,491$, USD 2018; $P<0.001$; Supplementary Figure 1, available in online article), equating to an incremental difference of over $\$ 500,000$. Inpatient costs accounted for the vast majority of these costs: $\$ 1.02$ million vs $\$ 576,581$ $(P<0.001)$, followed by outpatient and emergency costs. The total direct medical costs were largely driven by costs associated with complications, which remained significantly higher in patients with SR aGVHD (\$868,966 vs $\$ 396,757$; $\mathrm{P}<0.001)$. PPPM medical costs were also significantly higher by $\$ 38,130(\mathrm{P}<0.01)$.

Of the HSCT-related complications, SR aGVHD patients incurred significantly higher health care costs than controls relating to anemia, neutropenia, thrombocytopenia, and infection, as shown in Table 3 ( $\mathrm{P}<0.05$ for all comparisons).

Mean costs for ER visits were approximately twice as high in patients with SR aGVHD than controls (\$1,979 vs $\$ 826$; $P=0.033$ ), as were the majority of outpatient management costs, except for durable medical equipment costs, which were higher in the control cohort $(P<0.05$ for all; Figure 1). Medication costs were not significantly different between the 2 cohorts over either the 12- or 24 -month follow-up period (approximately $\$ 8,900$ per patient in each cohort over 12 months)

Total medical costs and costs for outpatient management, ER visits, and HSCT-related complications remained significantly higher in SR aGVHD patients than controls over the 24-month follow-up period $(\mathrm{P}<0.05$ for all comparisons; Supplementary Table 10, available in online article). 


\section{TABLE 3} Post-HSCT Complication Costs Over
12 Months in Pediatric Patients
(<18 Years) with SR aGVHD vs Controls
Without GVHD (USD, 2018 Cost Year)

\begin{tabular}{l|c|c|c}
\hline & $\begin{array}{c}\text { SR aGVHD } \\
\mathbf{n = 3 8}\end{array}$ & $\begin{array}{c}\text { Control } \\
\text { (no GVHD) } \\
\mathbf{n = 1 8 4}\end{array}$ & \multicolumn{1}{|c}{} \\
\cline { 2 - 3 } & Mean & Mean & P value \\
\hline All complications & $\$ 868,966$ & $\$ 396,757$ & $<0.001$ \\
\hline Anemia total & $\$ 411,470$ & $\$ 142,913$ & $<0.001$ \\
\hline Drug induced & $\$ 176,753$ & $\$ 44,818$ & $<0.001$ \\
\hline Nondrug induced & $\$ 250,433$ & $\$ 106,338$ & 0.006 \\
\hline Neutropenia total & $\$ 171,799$ & $\$ 58,886$ & 0.002 \\
\hline Drug induced & $\$ 30,014$ & $\$ 10,314$ & 0.175 \\
\hline Nondrug induced & $\$ 141,815$ & $\$ 48,579$ & 0.006 \\
\hline Thrombocytopenia total & $\$ 97,106$ & $\$ 36,221$ & 0.011 \\
\hline Liver diseases & $\$ 26,904$ & $\$ 37,311$ & 0.780 \\
\hline Gl diseases & $\$ 86,483$ & $\$ 48,425$ & 0.130 \\
\hline Infections & $\$ 542,286$ & $\$ 202,125$ & $<0.001$ \\
\hline
\end{tabular}

Note: Complication cost categories are not mutually exclusive. Differences in mean costs were modeled using a generalized linear model with gamma distribution and log-link, weighted for follow-up time.

aGVHD=acute graft-versus-host disease; $\mathrm{HSCT}=$ hematopoietic stem cell transplant; $S R=$ steroid refractory; $U S D=U S$ dollars.

Among patients with SR aGVHD, mean total costs were higher by about $\$ 1.8$ million for those who died compared with those who were alive within 12 months (Figure 2). Among the control cohort without GVHD, mean total costs were also higher for patients who died, with a difference of about $\$ 298,000$. Mean total costs were significantly higher for the SR aGVHD cohort vs the control cohort among the subgroup that died $(\mathrm{P}=0.002)$ and among those who survived for 1 year $(P=0.014)$.

\section{Discussion}

aGVHD in pediatric patients is a serious and life-threatening complication of allo-HSCT that often occurs within 100 days of transplantation. ${ }^{4,8}$ It is associated with substantial morbidity, decreased quality of life, and increased risk of mortality. ${ }^{6,8,9}$ Over half of patients with aGVHD do not respond to first-line treatment with steroids, yet there is no consensus on the second-line treatment of SR aGVHD. ${ }^{6,7,14-}$ ${ }^{16}$ Furthermore, the economic burden of aGVHD is high, yet there is little evidence of the economic impact of SR aGVHD on pediatric patients. A single-cohort retrospective study evaluated costs and HCRU among pediatric patients with aGVHD and found increased inpatient days and costs for patients with SR aGVHD relative to those with steroidresponsive aGVHD. ${ }^{20}$ We believe that our retrospective study of commercially insured US patients aged less than 18 years with SR aGVHD is the first to assess economic burden and HCRU in the pediatric SR aGVHD population across multiple treatment centers.

Our findings showed that pediatric patients with SR aGVHD incurred significantly higher HCRU and direct medical costs within the 12 months following allo-HSCT than controls who had no claims for GVHD. These results are consistent with recent findings on economic burden and HCRU among adult US patients with GVHD, which analyzed a cohort of patients with SR/high risk GVHD and found significantly longer median lengths of stay, higher median total costs, higher readmission rates, and higher inpatient mortality vs patients who underwent transplant but did not develop GVHD. ${ }^{21}$ Similarly, a retrospective review of adult patients in the United Kingdom found that patients with severe (grade 3/4) GVHD had higher readmission rates, mean number of inpatient days, and total cost of treatment relative to those with grade 1/2 GVHD. Overall, patients with GVHD had higher HCRU and costs than those without GVHD. ${ }^{23}$

Hematologic, GI, and infectious complications occurred more frequently in patients with SR aGVHD than controls (Table 2). Correspondingly, SR aGVHD patients had higher HCRU, hospital readmission rates (89\% vs $60 \%$; $P<0.001)$, outpatient parenteral nutrition rates (39\% vs $20 \%$; $P<0.01)$, and longer inpatient stay (by 31.6 days) compared with controls.

Of the SR aGVHD cohort, nearly all patients (95\%) escalated to second-line therapy with traditional immunosuppressants or biologics. A number of biologics were used in the second line (Supplementary Table 7, available in online article), and the variation in their use in clinical practice seen in this study underscores the lack of consensus on the second-line treatment of SR aGVHD., ${ }^{7,14-16}$

During the 12 months after HSCT, total direct medical costs for patients with SR aGVHD were significantly higher and nearly double that of controls $(\$ 1,212,944$ vs $\$ 673,491$; $\mathrm{P}<0.001)$. Medical costs for SR aGVHD were driven by post-HSCT complications, which accounted for $\$ 868,966$ of costs. When assessed by complication type, significantly higher costs were seen for anemia and infection (Table 3), which reflects the higher rate of these complications reported in SR aGVHD patients and may result from current second-line agents used. The rate of complications, HCRU, and medical costs seen over 24 months were consistent with those observed over 12 months. 


\section{FIGURE 1 Outpatient Costs Over 12 Months After HSCT in Pediatric Patients (<18 Years) with SR aGVHD vs Controls Without GVHD (USD, 2018 Cost Year)}
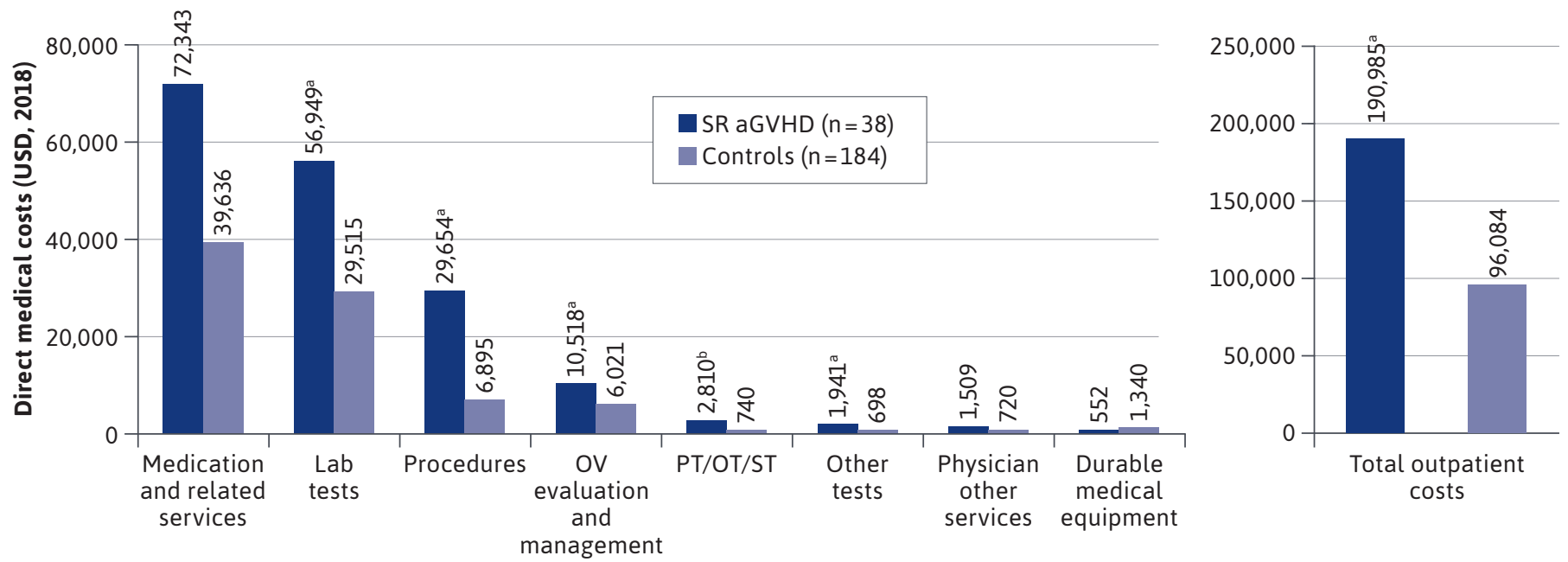

Note: Differences in mean costs were modeled using a generalized linear model with gamma distribution and log-link, weighted for follow-up time.

${ }^{a} P<0.05$.

${ }^{b} P<0.01$.

aGVHD = acute graft-versus-host disease; $\mathrm{HSCT}=$ hematopoietic stem cell transplant; $\mathrm{OV}=$ office visits; $\mathrm{PT} / \mathrm{OT} / \mathrm{ST}=$ physical therapy/occupational therapy/speech therapy; $S R=$ steroid refractory; USD = US dollars.

This study also found that costs are very high for patients with a poor outcome in SR aGVHD. Within 12 months of transplant, the costs incurred by patients with SR aGVHD who ultimately died were substantially higher (mean cost difference $\sim \$ 1.8$ million, $P=0.014$ ) than for those who survived. This difference was far less pronounced among the control cohort, in which a mortality outcome was associated with a mean total cost of about $\$ 298,000$ more than for those who survived.

\section{LIMITATIONS}

This study is subject to several limitations. First, we are not aware of validated algorithms to identify steroid-refractory aGVHD patients in claims. Our study definition is consistent with clinical literature; however, we did not conduct validation. Second, our analyses were strictly exploratory; for example, the presence of confounding by baseline characteristics was not examined. Third, data were derived from medical and pharmacy claims, which may have contained undetected coding errors. Moreover, all patients included in the study were enrolled in US commercial health insurance plans and satisfied all the inclusion/exclusion criteria. The results may not be generalizable to patients not selected, with other types of health insurance or who are uninsured, or to those outside the United States.
Another limitation of this work is that the comparator cohort was composed of patients who underwent HSCT and did not have any GVHD claims, rather than patients with steriod-responsive aGVHD. However, given that steroid responsiveness is generally established within 1 week of treatment and based on the relatively low cost of steroids, the cost burden of steriod-responsive aGVHD is likely to be only modestly higher than that of patients undergoing HSCT who do not develop GVHD. Some patients may have prolonged steroid treatment for mild to moderate symptoms of aGVHD, but this treatment is not likely to incur substantial additional costs. Thus, our reported cost difference between the cohorts of patients with SR aGVHD and no GVHD is likely to represent the upper range of the actual cost difference between patients with steroid-responsive and SR aGVHD. Because this was an exploratory study, future research could focus on developing an algorithm to identify steroid-responsive patients.

\section{Conclusions}

Our study indicates that within 1 year after HSCT, pediatric patients who develop SR aGVHD incur over half a million dollars in medical costs beyond costs incurred by those 


\section{FIGURE 2 Mean Total Per-Patient Medical Costs for Patients With or Without All-Cause Mortality Within 12 Months}

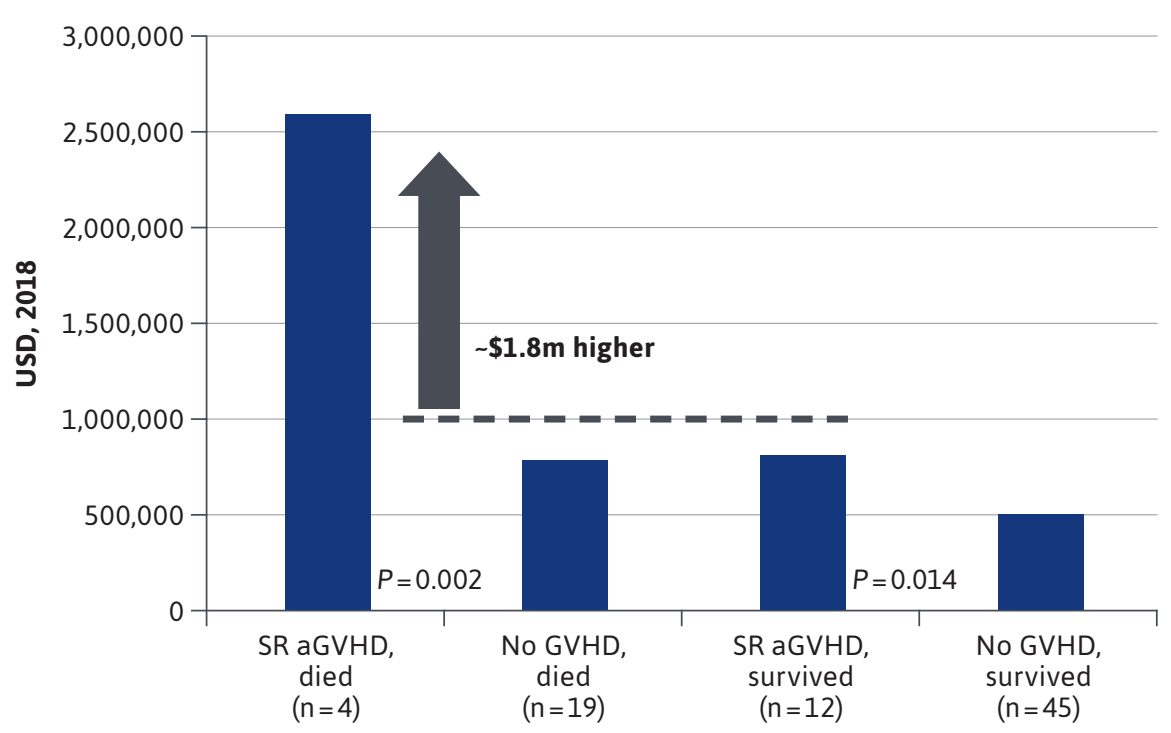

aGVHD = acute graft-versus-host disease; $S R=$ steroid refractory.

who do not develop GVHD, a difference largely driven by complications. Likewise, complication rates after HSCT and HCRU are higher in patients with SR aGVHD. These findings help to explain the high economic burden of pediatric patients with SR aGVHD and will help researchers to assess the value of novel therapeutic options for pediatric aGVHD.

\section{DISCLOSURES}

This research was sponsored by Mesoblast, Inc. Grabner is an employee of HealthCore, Inc., which acted as consultants to Mesoblast, Inc., during the conduct of this research. Strati is an employee of Mesoblast, Inc. Sandman and Forsythe are employees of Purple Squirrel Economics, which acted as consultants to Mesoblast, Inc., during the conduct of this research.

This work was presented at the AMCP Annual Meeting online in April 2020 and was an encore presentation at AMCP Nexus 2020 Virtual in October 2020.

\section{REFERENCES}

1. Passweg JR, Baldomero H, Basak GW, et al. The EBMT activity survey report 2017: a focus on allogeneic HCT for nonmalignant indications and on the use of non-HCT cell therapies. Bone Marrow Transplant. 2019;54(10):1575-85. doi:10.1038/s41409-019-0465-9

2. Khandelwal P, Millard HR, Thiel E, et al. Hematopoietic stem cell transplant activity in pediatric cancer between 2008 and 2014 in the United States: a CIBMTR report. Biol Blood Marrow Transplant. 2017;23(8):1342-49. doi:10.1016/j. bbmt.2017.04.018

3. D'Souza A, Fretham C. Current use and outcome of hematopoietic stem cell transplantation: CIBMTR summary slides, 2020. Accessed April 4, 2021. https:// www.cibmtr.org/ReferenceCenter/ SlidesReports/SummarySlides/Pages/ index.aspx

4. Jacobsohn DA, Vogelsang GB. Acute graft versus host disease. Orphanet J Rare Dis. 2007;2:35. doi:10.1186/1750-1172-2-35
5. Ball LM, Egeler RM. Acute GvHD: pathogenesis and classification. Bone Marrow Transplant. 2008;41(2):S58-S64. doi:10.1038/bmt.2008.56

6. Ruiz P. Pediatric graft versus host disease. Jyonouchi H, ed. MedScape. June 3, 2019. Accessed October 2, 2019. https://emedicine.medscape.com/ article/886758-overview\#showall

7. Dignan FL, Clark A, Amrolia P, et al. Diagnosis and management of acute graft-versus-host disease. Br J Haematol. 2012;158(1):30-45. doi:10.1111/j.1365-2141.2012.09129.x

8. Filipovich AH, Weisdorf D, Pavletic S, et al. National Institutes of Health consensus development project on criteria for clinical trials in chronic graftversus-host disease: I. Diagnosis and staging working group report. Biol Blood Marrow Transplant. 2005;11(12):945-56. doi:10.1016/j.bbmt.2005.09.004

9. Tomaras D, Strati E, Forsythe A. PF718 The economic and humanistic burden of graft-versus-host-disease (GVHD): a systemic literature review. Presented at: 24th Congress of the European Hematology Association; June 14, 2019; Amsterdam, Netherlands. Accessed April 8, 2021. https://journals.lww.com/ hemasphere/Abstract/2019/06001/ PF718_THE_ECONOMIC_AND_ HUMANISTIC_BURDEN_OF.613.aspx

10. Center for International Blood and Marrow Transplant Research. Transplant activity report covering 2013-2017.

Table 19. 2018. Accessed March 4, 2020. https://bloodstemcell.hrsa.gov/sites/ default/files/bloodstemcell/data/ transplant-activity/transplants-year-cellsource-age-donor.pdf

11. Zecca M, Pagliara D, Fagioli F, et al. Steroid-refractory acute GVHD in children: retrospective analysis of the AIEOP HSCT Registry. Blood. 2018;132(Suppl 1):4578-78. doi:10.1182/ blood-2018-99-114862

12. Westin JR, Saliba RM, De Lima M, et al. Steroid-refractory acute GVHD: predictors and outcomes. Adv Hematol. Published online November 3, 2011. doi:10.1155/2011/601953 
13. Arai S, Margolis J, Zahurak M, Anders V, Vogelsang GB. Poor outcome in steroidrefractory graft-versus-host disease with antithymocyte globulin treatment. Biol Blood Marrow Transplant. 2002;8(3):15560. doi:10.1053/bbmt.2002.v8.pm11939605

14. Knobler R, Berlin G, Calzavara-Pinton P, et al. Guidelines on the use of extracorporeal photopheresis. J Eur Acad Dermatol Venereol. 2014;28:1-37. doi:10.1111/jdv.12311

15. Martin PJ, Rizzo JD, Wingard JR, et al. First- and second-line systemic treatment of acute graft-versus-host disease: recommendations of the American Society of Blood and Marrow Transplantation. Biol Blood Marrow Transplant. 2012;18(8):115063. doi:10.1016/j.bbmt.2012.04.005

16. Ruutu T, Gratwohl A, de Witte T, et al. Prophylaxis and treatment of GVHD: EBMT-ELN working group recommendations for a standardized practice. Bone Marrow Transplant. 2014;49(2):168-73. doi:10.1038/bmt.2013.107

17. Jakafi. Prescribing information. Incyte Corporation; 2019. Accessed September 30, 2019. https://www.jakafi.com/pdf/ prescribing-information.pdf
18. Yu J, Parasuraman S, Shah A, Weisdorf D. Mortality, length of stay and costs associated with acute graftversus-host disease during hospitalization for allogeneic hematopoietic stem cell transplantation. Curr Med Res Opin. 2019;35(6):983-88. doi:10.1080/03007995. 2018.1551193

19. Yu J, Lal L, Anderson A, DuCharme M, Parasuraman S, Weisdorf DJ. Healthcare resource utilization (HCRU) and costs associated with acute graft-vs-host disease (aGVHD) following allogeneic hematopoietic stem cell transplant (a-HSCT): a retrospective claims database analysis. Biol Blood Marrow Transplant. 2018;24(3):S189-S190. doi:10.1016/j. bbmt.2017.12.145

20. Ricci A, Jin Z, Broglie L, et al. Healthcare utilization and financial impact of acute-graft-versus host disease among children undergoing allogeneic hematopoietic cell transplantation. Bone Marrow Transplant. Published online September 19, 2019. doi:10.1038/ s41409-019-0688-9
21. Yu J, Judy JT, Parasuraman S, Sinha M, Weisdorf D. Inpatient healthcare resource utilization, costs, and mortality in adult patients with acute graft-versus-host disease, including steroid-refractory or high-risk disease, following allogeneic hematopoietic cell transplantation. Biol Blood Marrow Transplant. 2020;26(3):60005. doi:10.1016/j.bbmt.2019.10.028

22. US Bureau of Labor Statistics. Measuring price change in the CPI: medical care. March 20, 2020. Updated November 25, 2020. Accessed April 28, 2020. https://www.bls.gov/cpi/factsheets/medical-care.htm

23. Dignan FL, Potter MN, Ethell ME, et al. High readmission rates are associated with a significant economic burden and poor outcome in patients with grade III/IV acute GvHD. Clin Transplant. 2013;27(1):E56-E63. doi:10.1111/ctr.12065 